\title{
2018 annual report of thoracic surgery service at Shanghai Chest Hospital
}

\author{
Yiyang Wang", Jizhuang Luo", Yu Yang", Xinghua Cheng", Tianxiang Chen", Feng Yao, Zhitao Gu, \\ Yifeng Sun, Qingqian Luo, Zhigang Li, Jun Yang, Teng Mao, Shijie Fu, Rui Wang, Jie Zhang, Heng Zhao, \\ Yongchun Yu, Changqing Pan, Wentao Fang
}

Department of Thoracic Surgery, Department of Pulmonary Oncology, Shanghai Chest Hospital, Jiaotong University Medical School, Shanghai 200025, China

Contributions: (I) Conception and design: W Fang; (II) Administrative support: Y Yu, C Pan; (III) Provision of study materials or patients: F Yao, Q Luo, Z Li, J Yang, T Mao, J Zhang, H Zhao, W Fang; (IV) Collection and assembly of data: X Cheng, T Chen, F Yao, Z Gu, Y Sun, S Fu, R Wang; (V) Data analysis and interpretation: Y Wang, J Luo, Y Yang, F Yao, W Fang; (VI) Manuscript writing: All authors; (VII) Final approval of manuscript: All authors.

\#The authors contributed equally to this work.

Correspondence to: Wentao Fang, MD, Professor. Department of Thoracic Surgery, Shanghai Chest Hospital Jiaotong University Medical School, Shanghai 200025, China. Email: vwtfang12@shchest.org.

Background: The Thoracic Surgery team at the Shanghai Chest Hospital has published its first annual report since 2017, which mainly contained the key achievements in the year before. This second annual report reviewed the efforts and achievements of the Thoracic Surgery service in the year 2018. Continuous effort has been made to further improve our service.

Methods: Patients received thoracic surgeries in 2018 at the Department of Thoracic Surgery and the Department of Thoracic Oncological, Shanghai Chest Hospital, were retrospectively collected. Detailed data on surgical volume, procedures, and peri-operative outcomes, stratified by each specialty, were carefully studied and compared with the results from previous years. Progress in education and research were also reported.

Results: In 2018, 58 thoracic surgeons, including 23 attending surgeons and 35 clinical fellows and residents, performed 14,054 major thoracic surgeries, including 11,958 pulmonary procedures, 866 esophageal procedures, 957 mediastinal procedures, 45 trachea procedures, and 7 lung transplantations. The total volume increased by $13.9 \%$ compared with the previous year. Among them, $88.4 \%$ were accomplished via minimally invasive approaches, including both video-assisted thoracoscopic/laparoscopic surgery and robotic surgery. There was a clear trend towards increased use of minimally invasive techniques for more complicated thoracic diseases in each specialty. More importantly, the unplanned reoperation rate, readmission to ICU rate, and 30-day mortality rate were all maintained in an extremely low level, being $0.37 \%, 0.82 \%$ and $0.21 \%$, respectively.

Conclusions: Tremendous effort has been engaged in improving Thoracic Surgery service at the Shanghai Chest Hospital. The year 2018 witnessed not only continuous increase in volume but also persistent high quality and safety in patient care. With these accomplishments and focus on more innovations, we will strive to deliver higher level of medical services in the future.

Keywords: Thoracic surgery; minimally invasive surgery; surgical volume; mortality

Received: 09 December 2019; Accepted: 28 December 2019; Published: 31 December 2019.

doi: $10.21037 /$ shc.2019.12.07

View this article at: http://dx.doi.org/10.21037/shc.2019.12.07 


\section{Introduction}

Thoracic Surgery Service at the Shanghai Chest Hospital was founded by famous Chinese cardiothoracic surgeons Dr. Huang Jiasi and Dr. Gu Kaishi in 1957. From the very beginning, it has committed to deliver state-of-theart surgical treatment, innovative scientific research and rigorous surgical education from generations to generations. Currently, thoracic surgery service at the Shanghai Chest Hospital consists of the Department of Thoracic Surgery and the Department of Surgical Oncology. It is one of the largest thoracic surgery centers in China and enjoys high reputation for its full range of subspecialties (including lung, esophagus, mediastinum, trachea and lung transplantation), high volume of thoracic surgeries and the ability to treat all kinds of complex, difficult and severe chest diseases.

Currently, thoracic surgery service at the Shanghai Chest Hospital has 405 beds in six wards and 27 beds in ICU. Volume of thoracic surgery has been expanding steadily, with a two-digit percentage year-on-year increase throughout the past decade. In 2018, 58 surgeons, including 23 attending surgeons and 35 clinical fellows and residents performed 14,054 cases of thoracic surgeries, including 11,958 pulmonary procedures, 866 esophageal procedures, 957 mediastinal procedures, 45 trachea procedures, and 7 lung transplantations, increased by $13.9 \%$ compared with the previous year (Figure 1).

The Shanghai Chest thoracic surgery team is devoted to reducing surgical trauma and enhancing recovery after surgery by using minimally invasive techniques including both video-assisted and robotic-assisted thoracic surgery (VATS and RATS), especially for patients of senile age, with poor cardiopulmonary function, or after neoadjuvant therapy. In 2018, the proportion of minimally invasive surgery has further increased to $88.4 \%$, with a total of 12,430 major thoracic procedures accomplished via minimally invasive approaches. Noticeably, the singleinstitution number of RATS thoracic procedures has remained at the top worldwide for many years. The Shanghai Chest thoracic surgery team has successfully carried out RATS and uniportal VATS sleeve lung resection, VATS/RATS segmentectomy, VATS/RATS esophagectomy, VATS pneumonectomy and VATS resection and reconstruction of carina and trachea, as distinct features of the Shanghai Chest. With increasing focus on surgical indication and quality control, high accuracy of diagnosis and low mortality rate has been maintained, and optimal perioperative results have been maintained through the years. Unplanned reoperation rate, readmission to the ICU rate and 30-day mortality rate were merely $0.37 \%, 0.82 \%$ and $0.21 \%$, respectively, in 2018 (Figure 2).

\section{Methods}

\section{Materials}

We enrolled patients receiving surgical resections at Department of Thoracic Surgery and Department of Oncological Surgery of Shanghai Chest Hospital from January 1st, 2018 to December 31th, 2018. Patients would be reclassified into different surgical divisions including pulmonary, esophageal, mediastinal, tracheal, and lung transplantation divisions according to different diseases and surgical treatments. Volumes of surgery, various types of surgical procedures, disease types, intraoperative and postoperative clinical outcomes and other characteristics were retrospectively collected.

\section{Statistical analysis}

Categorical variables were presented as frequencies (percentages). Continuous variables were plotted as mean \pm standard deviation (SD) if necessary. Year-on-year figures were plotted when comparing results with the previous time period. Statistical analysis were conducted by using the SPSS software (version 22.0, USA).

\section{Results}

\section{Pulmonary Surgery Division}

In the year of 2018, a total of 11,958 pulmonary resections were carried out, increased by $13.9 \%$ compared to the year before (Figure 3). Of these patients, 11,423 cases received minimally invasive surgery $(95.5 \%)$, including 11,023 cases $(92.2 \%)$ of video-assisted thoracic surgery (VATS), 400 cases $(3.3 \%)$ of robotic-assisted thoracic surgery (RATS), and 535 cases $(4.5 \%)$ of open thoracotomy (Figure 4 ). Conversion rate to open thoracotomy was merely $0.7 \%$ (83 cases), with 23 events $(0.2 \%)$ of intraoperative massive hemorrhage during VATS/RATS procedures.

Of those 11,958 patients, 10,915 were confirmed as having malignancy and only 1,043 turned out to have benign diseases. The rate of diagnostic accuracy was as high as $91.3 \%$. The majority of pulmonary resections were lobectomy ( $\mathrm{n}=7,626,63.8 \%)$, followed by wedge resection $(n=2,803,23.4 \%)$, segmentectomy $(n=1,321,11.1 \%)$, sleeve 


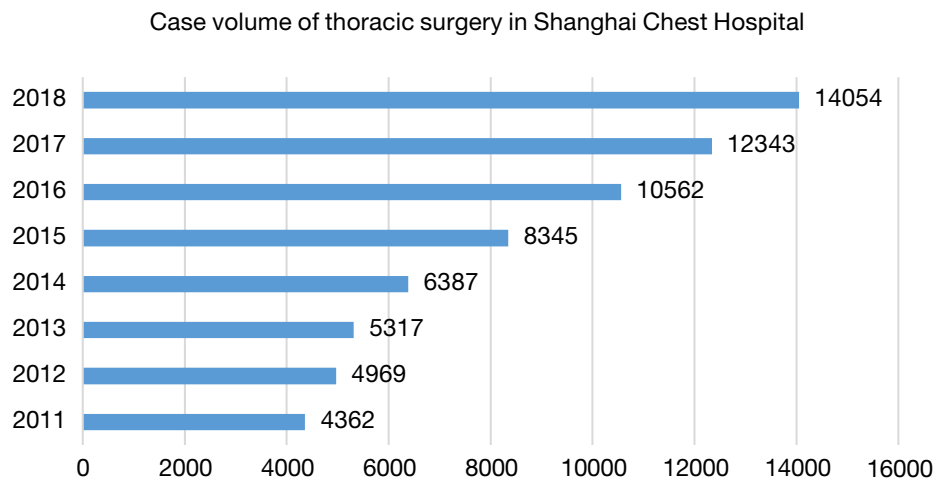

Figure 1 Case volume of thoracic surgery in Shanghai Chest Hospital.

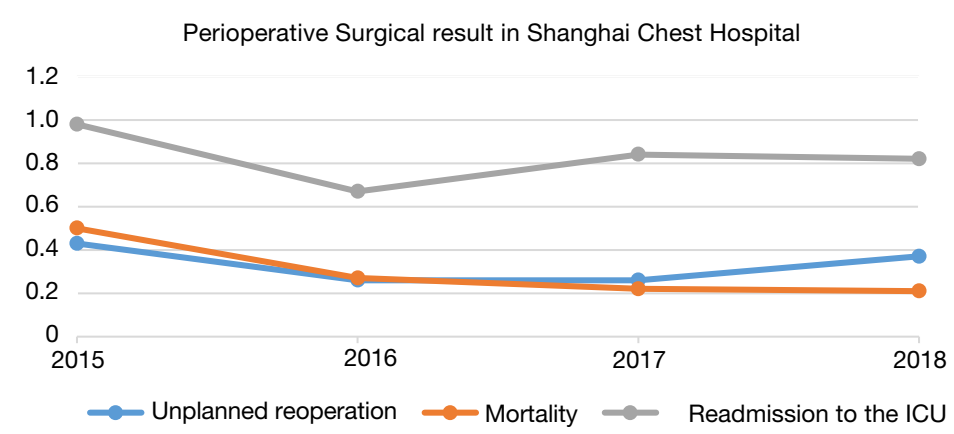

Figure 2 Perioperative surgical result in Shanghai Chest Hospital.

The total number of lung operations

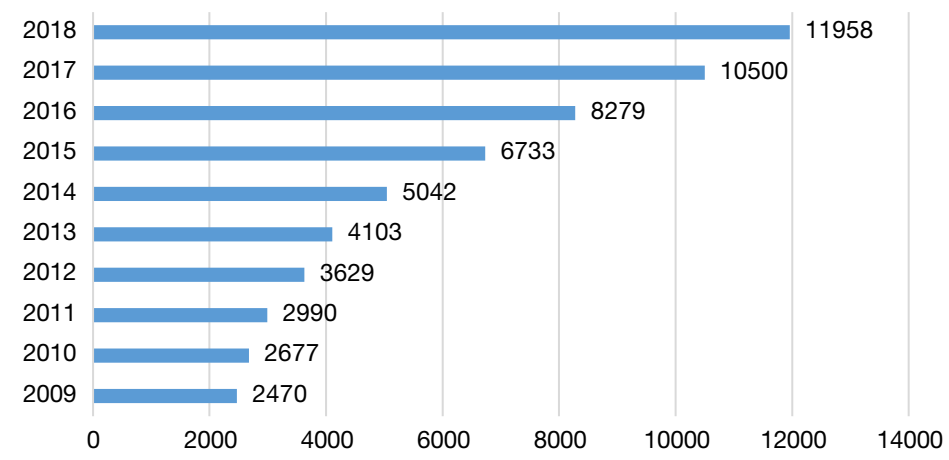

Figure 3 The total number of lung operations in Shanghai Chest Hospital.

resection $(\mathrm{n}=119,1.0 \%)$ and pneumonectomy $(\mathrm{n}=89,0.7 \%)$ (Figure 5). With the rapid increase in early stage lung cancers detected by CT screening, sublobar resections have been increasingly applied for small GGO containing lesion. Number of segmentectomies reached 1,321 in 2108, increased by $36.2 \%$ from 2017 , almost all of them were accomplished via minimally invasive approaches (Figure 6).
In addition to surgery for early stage diseases, there is also a remarkable increase in using minimally invasive surgical techniques to fulfill complicated pulmonary procedures for locally advanced disease. Among a total of 119 sleeve resections in 2018, 25\% of them were successfully done by VATS (Figure 7). What is more, 16 cases of VATS (18\%) was witnessed in a total of 87 pneumonectomies (Figure 8). 


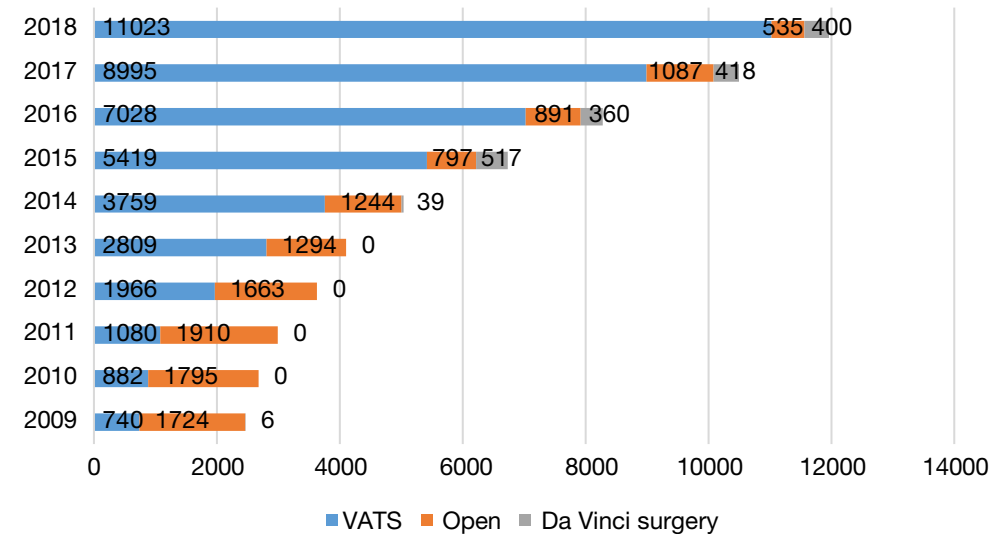

Figure 4 Case volume of three types of lung surgery carried out in Shanghai Chest Hospital.

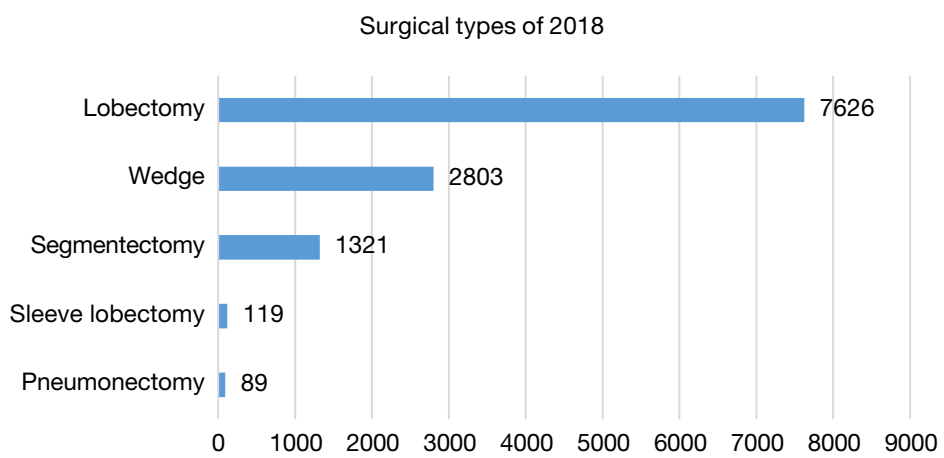

Figure 5 Surgical procedures of lung disease carried out in 2018.

\section{Esophageal Surgery Division}

In 2018, a total of 866 cases of esophageal surgery were performed, including 727 cases of esophageal cancers and 139 cases of benign esophageal diseases (Figure 9). Comparing to the year 2017, number of benign esophageal diseases has increased by $8.0 \%$ and number of esophageal cancers has increased by $4.5 \%$.

There were 139 patients diagnosed with benign esophageal disease underwent surgical treatment in 2018 (Figure 10), including 32 (23.0\%) cases of benign esophageal tumors, $15(10.8 \%)$ cases of gastroesophageal reflux disease, $19(13.7 \%)$ cases of hiatus hernia, $6(4.3 \%)$ cases of diverticulum, $6(4.3 \%)$ cases of achalasia, $8(5.8 \%)$ cases of tracheal-esophageal fistula, $8(5.8 \%)$ cases of esophageal stenosis and 45 (32.4\%) cases of other benign diseases.

Most of benign esophageal diseases were treated via minimally invasive approaches, with $22(15.8 \%)$ patients underwent endoscopic therapies, $66(47.5 \%)$ patients underwent thoracoscopic/laparoscopic assisted surgery, and $3(2.2 \%)$ patients underwent robot assisted procedures.

In 2018, a novel minimally invasive procedure [laparoscopic magnetic sphincter augmentation (MSA)] for gastroesophageal reflux disease has been tried at the Shanghai Chest Hospital. This MSA could increase the tension of the lower esophageal sphincter to achieve antireflux effect. Comparing to Nissen fundoplication, MSA can achieve better short-term surgical outcomes, especially in terms of gas-belching, due to shorter operative time and less complication, bloating or side-effects. As the first phase II MSA clinical trial in China, a total of 12 centers has joined the study, with an expected sample size of 100 cases and the final results expected in 2022. There were already six patients successfully treated by laparoscopic MSA at the Shanghai Chest Hospital in 2018, with a mean operative time of $32.6 \pm 6.2$ minutes. All patients were successfully discharged within 3 days after surgery.

In 2018, there were 727 esophageal cancer patients 


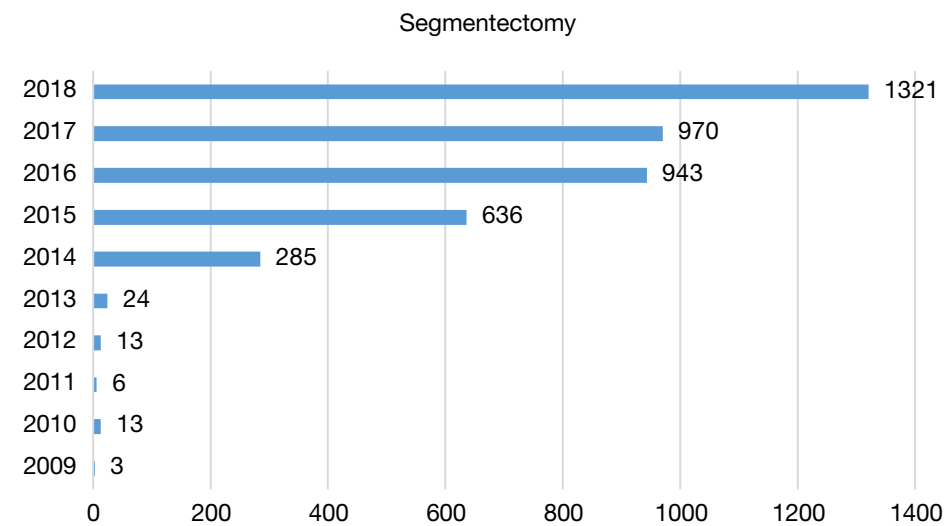

Figure 6 Case volume of segmentectomy carried out in Shanghai Chest Hospital.

Sleeve Lobectomy of 2018

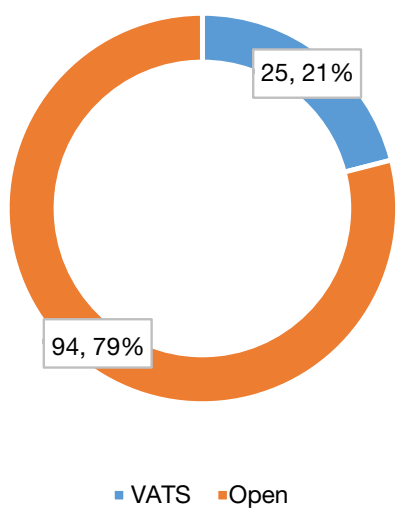

Figure 7 Case volume and surgical approaches for sleeve lobectomy carried out in 2018.

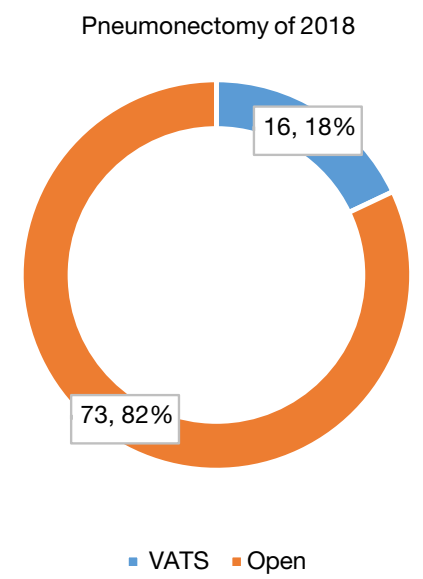

Figure 8 Case volume and surgical approaches for pneumonectomy carried out in 2018. undergone endoscopic or surgical treatment at the Shanghai Chest Hospital (Figure 11).

Endoscopic submucosal dissection (ESD) has becoming the first-line therapy for patients with early (mucosa and superficial submucosa) esophageal cancers. Sixty-five cases of ESD was completed in 2018, increased by $85.7 \%$ comparing to 2017 (Figure 12). Only one patient (1.6\%) had to be converted to esophagectomy due to perforation during ESD. The mean length of hospital stay was $4.0 \pm 3.2$ days and no other major complication was observed.

A total of 662 patients underwent esophagectomy, including 129 cases of open esophagectomy, 422 cases of video assisted esophagectomy and 111 cases of robot assisted esophagectomy. Minimally invasive esophagectomy (MIE) accounts for $80.5 \%$ in the entire cohort. In addition to 417 thoracoscopic assisted esophagectomies, singleport mediastinoscopic assisted transhiatal esophagectomy with en-bloc lymphadenectomy was successfully completed in 5 patients. McKeown is the most preferred procedure (543, 82.0\%), with 52 patients having left-thoracic Sweet procedure, 29 patients having Ivor-Lewis procedure, and the other 39 patients having transhiatal esophagectomy, laryngo-pharyngo-esophagectomy and so on.

Most patients had tumor located at the thoracic esophagus, with 50 having tumors located at the gastroesophageal junction and 25 located at the cervical esophagus. The most common tumor histology was squamous cell carcinoma $(580,87.6 \%)$, with $50(7.6 \%)$ adenocarcinomas, 17 (2.6\%) neuroendocrine carcinomas and 15 (2.3\%) other types of malignant tumors (Figure 13).

Challenging surgical techniques have also been increasingly used in 2018. For patients with tumors located at the upper 


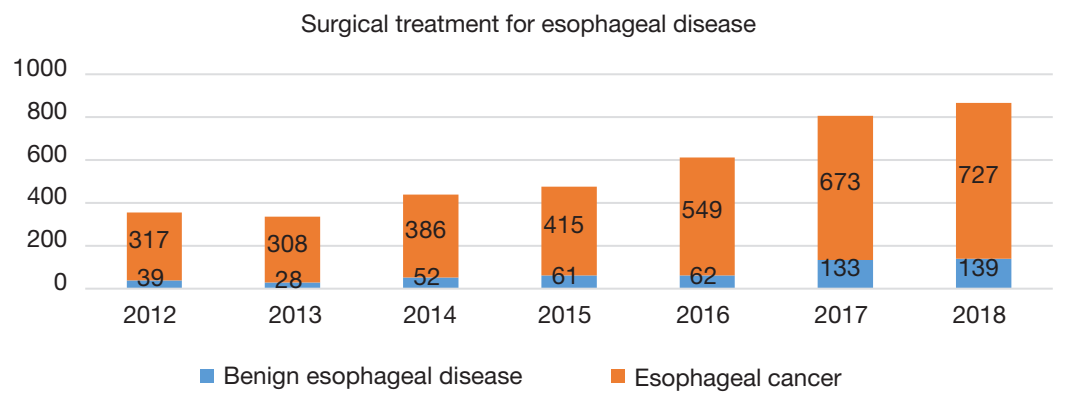

Figure 9 Case volume of esophagus disease and esophagus cancer surgery carried out in Shanghai Chest Hospital.

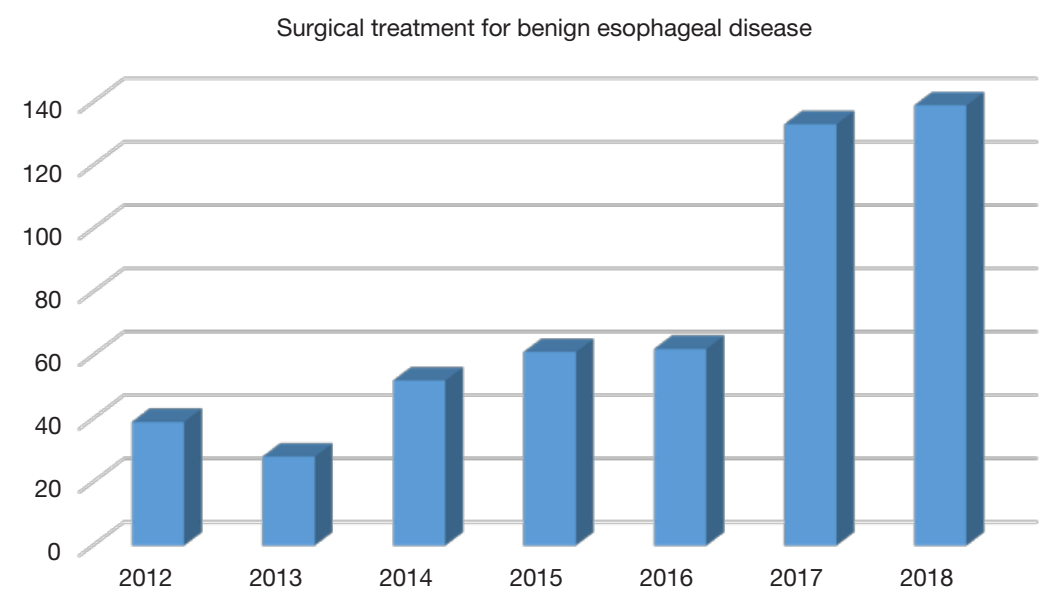

Figure 10 Surgical treatment for benign esophageal disease.

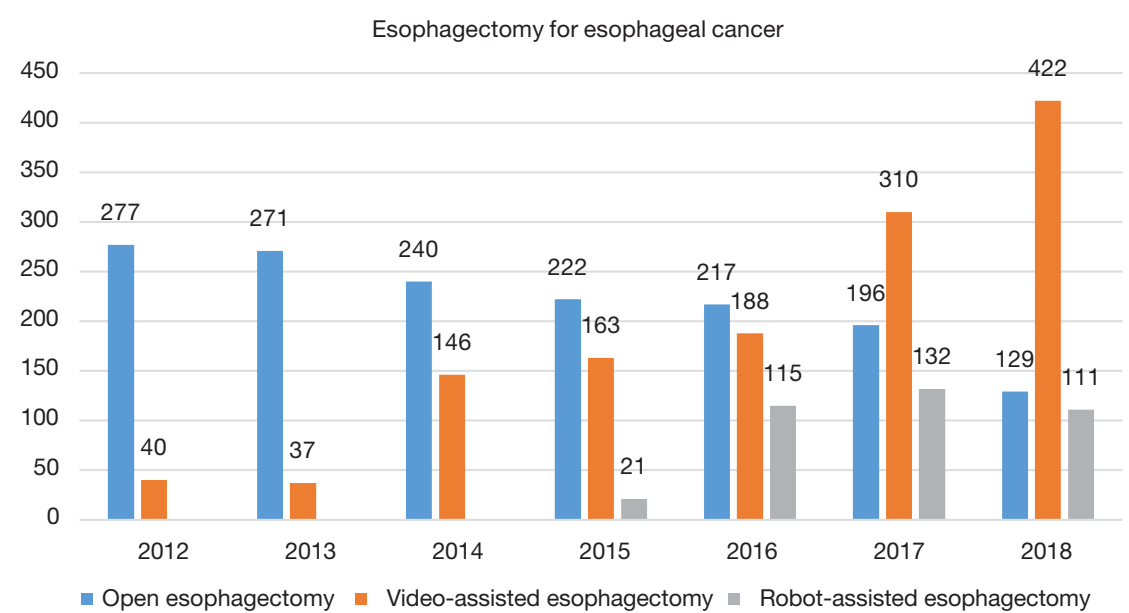

Figure 11 Esophagectomy for esophageal cancer in Shanghai Chest Hospital.

thoracic or cervical esophagus, 17 cases of total laryngopharyngo-esophagectomy (TPLE), 10 cases of laryngopreserving esophagectomy (LPE), and 2 cases of free jejunal interposition were performed. For patients who received definitive chemoradiation or endoscopic dissection and then experienced tumor progression, 17 salvage esophagectomies 


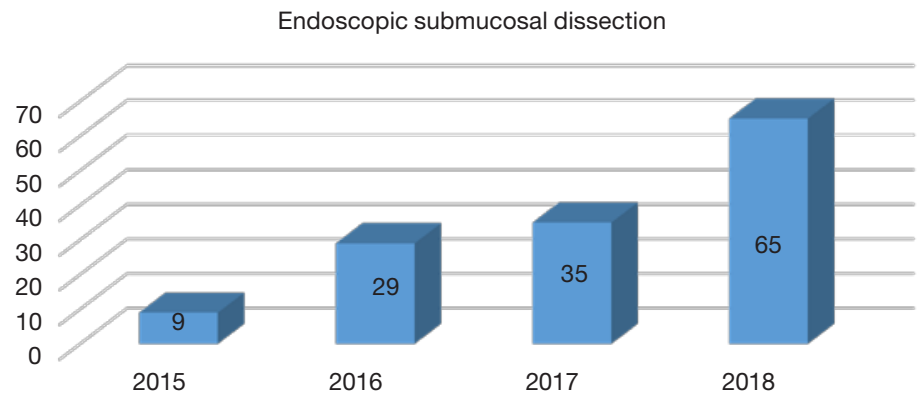

Figure 12 Endoscopic submucosal dissection for esophageal cancer in Shanghai Chest Hospital.

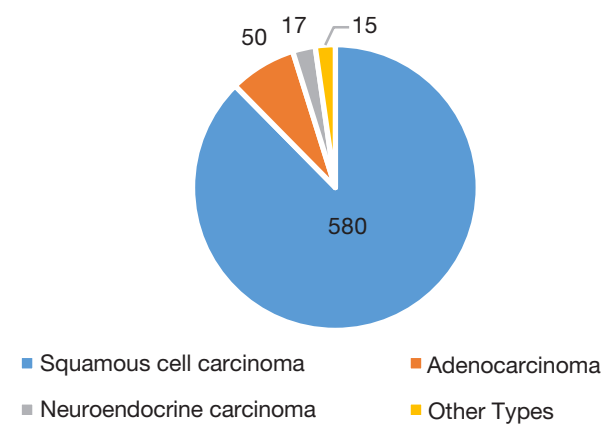

Figure 13 Histological type of 662 patients who received esophagectomy.

were successfully accomplished (Figure 14). For patients who had history of gastrectomy, 15 colon replacement were performed (Figure 15). The 30-day mortality rate was only $0.6 \%$, with a mean length of postoperative hospital stay of $13.6 \pm 10.3$ days. The postoperative complication rate was $34.5 \%$ for the entire cohort.

\section{Mediastinal Surgery Division}

There were altogether 957 patients with mediastinal diseases receiving surgical resection at the Shanghai Chest Hospital in 2018. Among them, 557 patients (56.5\%) underwent VATS procedure. Eighty patients $(8.1 \%)$ underwent RATS procedure, and 320 patients (32.5\%) underwent open surgery (Figure 16).

Histologically, there were 498 cases of thymic epithelial tumors (TETs), followed by 222 cases of cysts, 94 cases of neurogenic tumors, 44 cases of germ cell tumors, and 26 cases of lymphatic tumors (Figure 17). Among these 498 TETs, there were 373 thymomas (19 type A, 141 type $\mathrm{AB}, 194$ type B, 14 micronodular thymoma, 5 metaplastic thymoma), 109 thymic carcinomas and 16 neuroendocrine thymic tumors (NETT) (Figure 18). Among the 44 germ cell tumors, there were 30 mature teratomas, 3 immature teratomas, 4 seminomas, 6 yolk sac tumors and 1 mixed germ cell tumor (Figure 19).

There has been a rapid increase in number of complex anterior mediastinum procedures accomplished under VATS since 2010 (Figure 20), among them three patients with locally advanced thymic tumors invading superior vena cava received thymectomy together with tangential superior vena cava resection under VATS.

Number of complicated anterior mediastinum procedures via open surgery for locally advanced TETs (UICC $8^{\text {th }}$ TNM staging II-IV) continued to increase as well, mostly for recurrent tumors or those after induction therapies (Figure 21). Among the 122 patients (38\%) who received open procedure in 2018, 48 patients received induction therapy for TETs (39.3\%). Significantly, rate of preoperative therapy for stage III and IV TET patients remained as high as nearly $30 \%$ in recent years (Figure 22).

Clinical researches in mediastinal diseases continued to intervene with daily practice. These include the feasibility study of minimally invasive surgery for locally advanced TETs, surgical pleurectomy followed by hemithorax pleural irradiation for stage IVa TETs, induction sequential chemoradiation for potentially resectable stage IIIb-IV TETs, prospective observational study for lymph node dissection in TETs, and a phase II trial for a domestically developed novel anti-VEGF agent in late stage/recurrent thymic carcinomas.

\section{Tracheal Surgery Division}

Total number of major procedures completed in tracheal surgery over the last decade at the Shanghai Chest is highlighted in Figure 23. Altogether 45 cases of trachea 


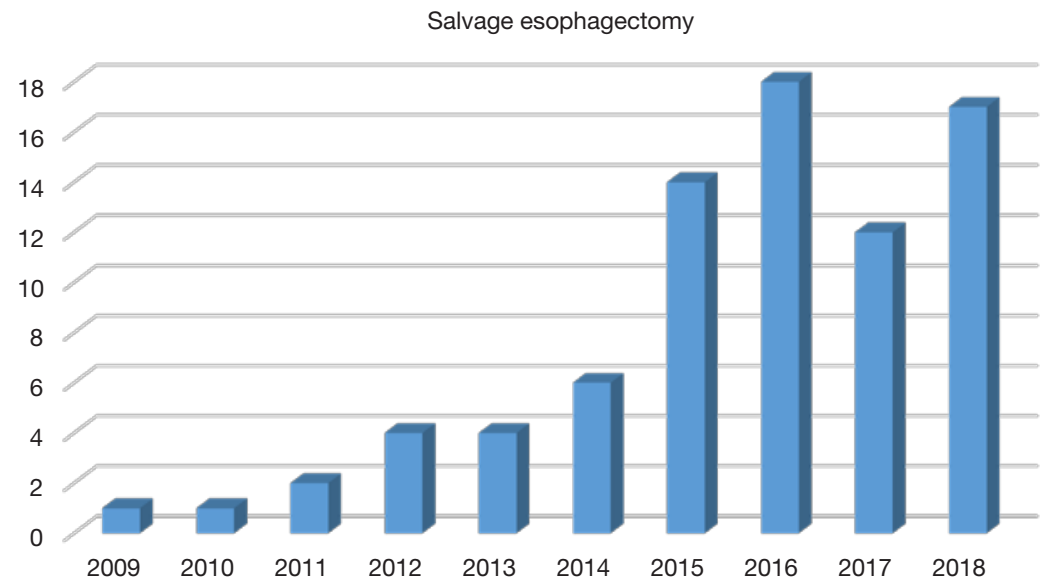

Figure 14 Salvage esophagectomy volume in Shanghai Chest Hospital.

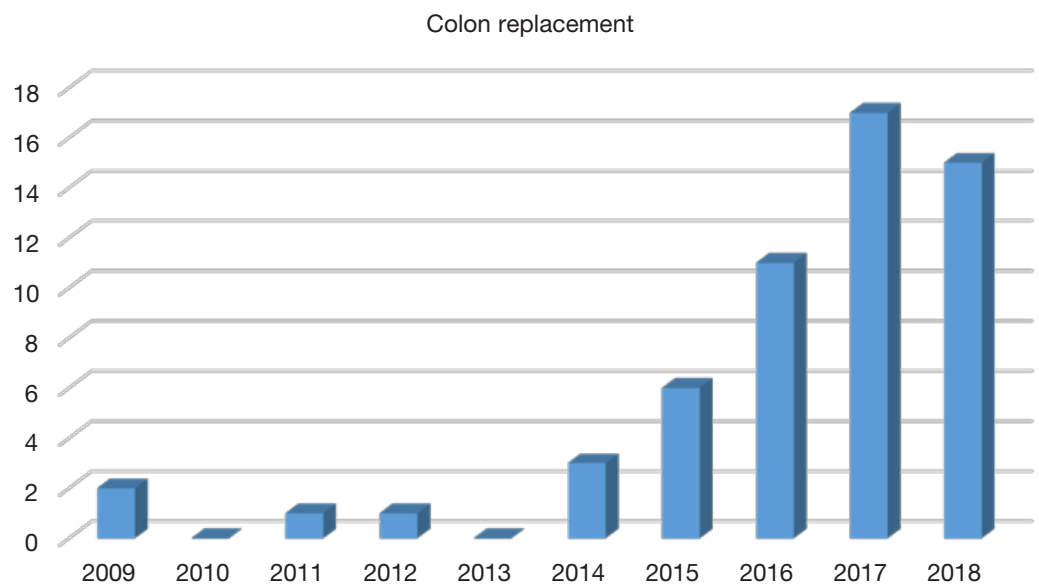

Figure 15 Colon replacement volume in Shanghai Chest Hospital.

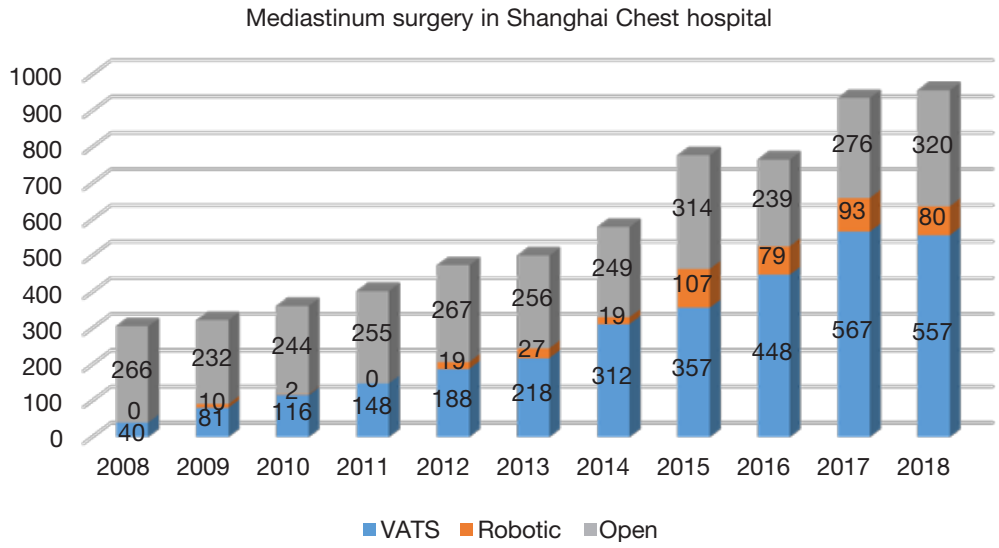

Figure 16 Mediastinum surgery in Shanghai Chest Hospital. 
and bronchial surgery were carried out in 2018, including 25 cases of trachea surgeries, 11 cases of main bronchial surgery and 9 cases of carina resection and reconstruction.

The disease spectrum included benign and malignant lesions in the trachea, main bronchi, or the carina, the majority of them being malignant tumors. Among the tracheal and main bronchial malignancies, the most common type was adenoid cystic carcinoma (18 cases), followed by 7 cases of squamous cell carcinoma, 4 cases of mucoepidermoid carcinoma and 3 cases of carcinoid. Only one case was a small cell carcinoma. The 12 cases of benign diseases included airway stenosis after trauma, neurilemmoma, inflammatory myofibroblastic tumor and glomus tumor (Figure 24).

Based on preoperative imaging results and bronchoscopy examination, right posterolateral thoracotomy and left

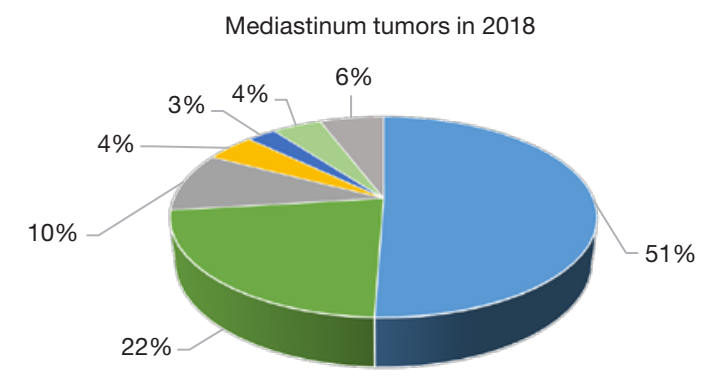

$$
\begin{aligned}
& \text { - Thymic tumor Germ - Cyst " Neurogenic tumor } \\
& \text { - cell tumor Other - - Lymphatic tumor - Other Malignant tumor } \\
& \text { - benign disease }
\end{aligned}
$$

Figure 17 Mediastinum tumors. posterolateral thoracotomy were adopted in 11 cases each. Cervical incision was used in 14 cases. Additional partial sternotomy was applied in 6 cases to ensure radical resection and to facilitate reconstruction. Sternotomy were adopted in two cases and robotic assisted approach was successfully performed in one case (Figure 25). Owing to difficult airways, three patients received surgery under extracorporeal circulation.

Remarkably, a robotic assisted carina resection and reconstruction was successfully accomplished last year at the Shanghai Chest. A 71-year-old gentleman came to us with irritative cough for three month. He had a 40 pack-year history of smoking and type II diabetes mellitus. Computed tomography showed a mass in the carina. Diagnosis of squamous cell carcinoma was confirmed by bronchoscopy. The patient underwent a robotic assisted resection and reconstruction of carina. His postoperative course was uneventful, and he was discharged on the 9th postoperative day. This is the first case ever reported showing the feasibility of robotic assisted resection and reconstruction of carina (Figure 26).

\section{Lung transplantation in 2018}

The first successful lung transplantation in Shanghai was accomplished at the Shanghai Chest Hospital in 2002. Last year, a book named 'Advances in Lung Transplantation' edited by the Shanghai Chest Hospital focusing on recent progress in the field of lung transplantation was published. In 2018, there were 7 patients registered in the waiting list of lung transplantation because of diffuse bronchiectasis

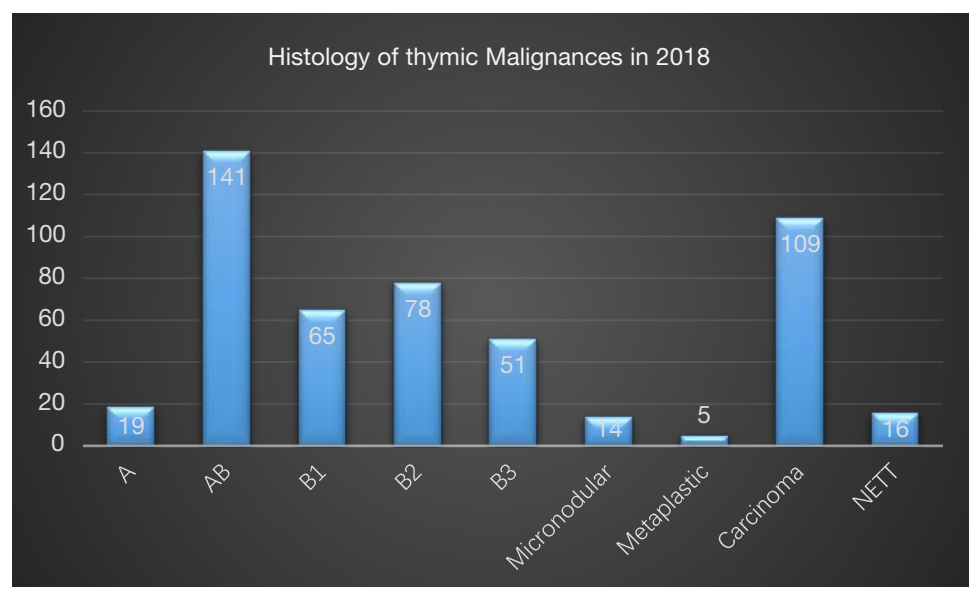

Figure 18 Histology of thymic malignances. 
( 2 cases), chronic pulmonary emphysema (1 case), pulmonary lymphangioleimyomatosis (1 case), idiopathic pulmonary fibrosis (2 cases) and obliterative bronchiolitis after lung transplantation ( 1 case). Among these patients, 4 cases received lung transplantation, including single lung transplantation, sequential double lung transplantation and retransplantation. So far there have been 4 cases of successful retransplantation in our center.

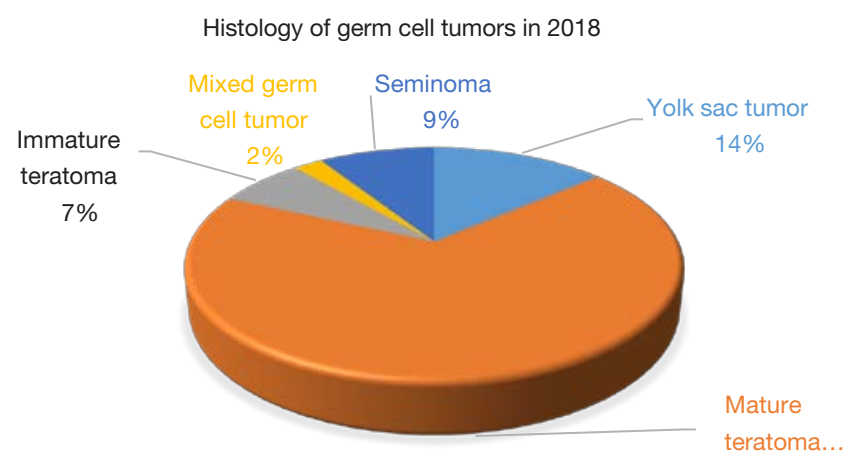

Figure 19 Germ cell tumors.

\section{Research and education}

Listed in Supplementary file 1 are the research activities fulfilled or still ongoing in 2018 in Thoracic surgery at the Shanghai Chest. Altogether there were 16 clinical trials and basic studies, most of them focusing on thoracic oncology. A total of 34 papers were accepted for publication in English literatures (Supplementary file 2).

In 2018, the National CME program on CardioThoracic Surgery and the international fellowship program at the Shanghai Chest has enrolled a total of 76 fellows from all regions of China and 8 international fellows from Europe and Asia. In the aspect of graduate education, there were $8 \mathrm{PhD}$ and $4 \mathrm{MS}$ students from the Medical college of Shanghai Jiao Tong University trained in the Department of Thoracic Surgery and the Department of Surgical Oncology last year. Other educational events are listed in Supplementary file 3. In addition, more than a dozen short-period immersion programs were hosted at the Shanghai Chest Hospital in the year 2018. And almost every weekend, there was a wet lab ongoing in minimally

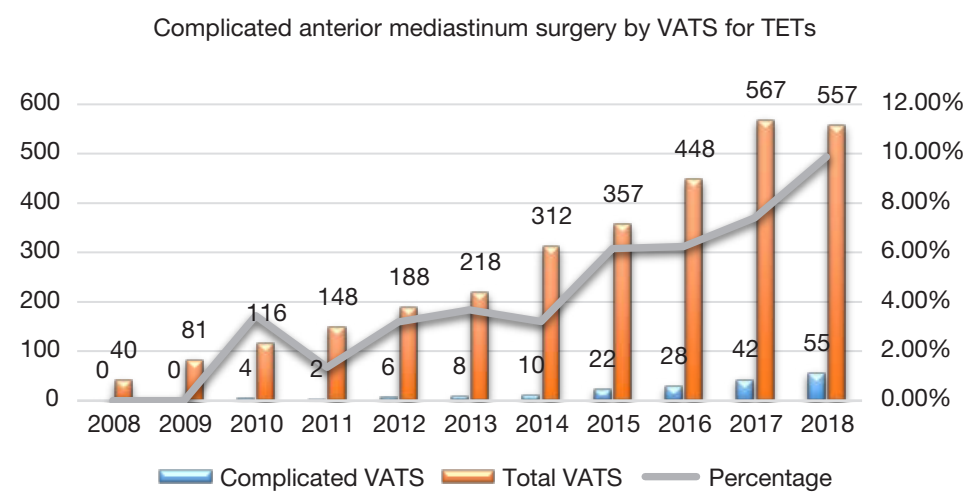

Figure 20 Complicated anterior mediastinum surgery by VATS for thymic tumors. VATS, video-assisted thoracic surgery.

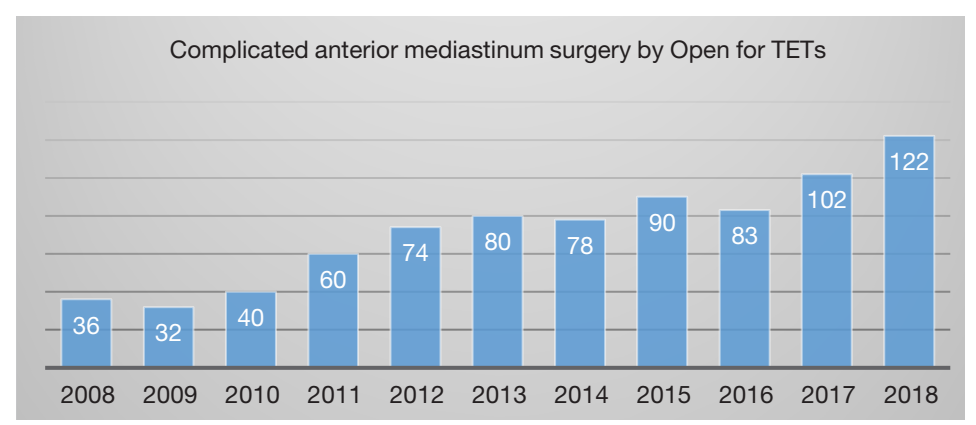

Figure 21 Complicated anterior mediastinum surgery by open for thymic tumors. 


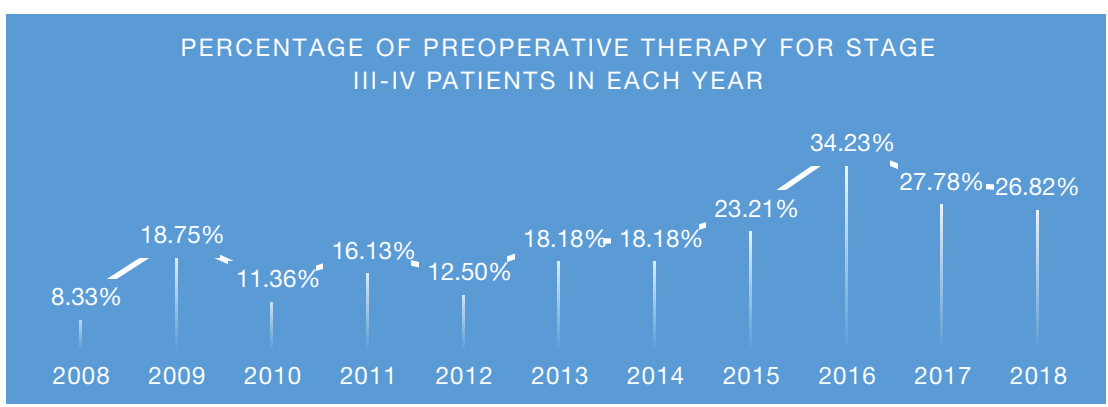

Figure 22 Percentage of preoperative therapy for stage III-IV patients in each year.

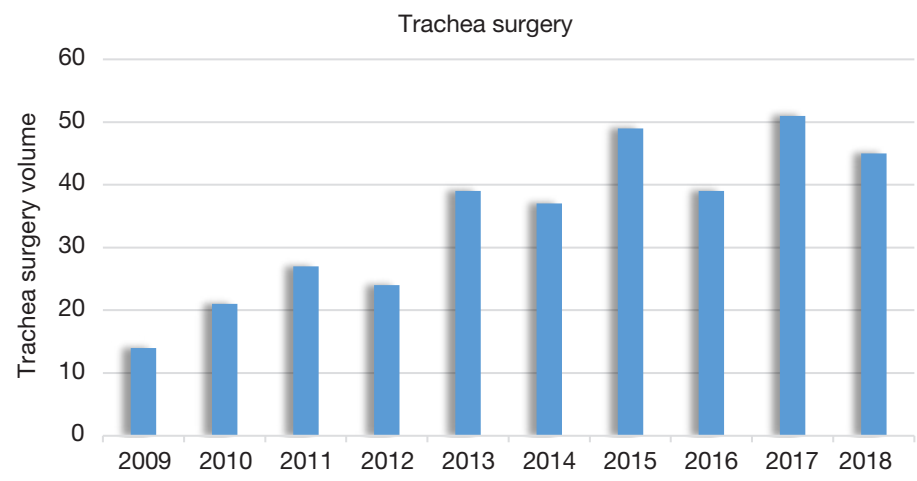

Figure 23 Trachea surgery in Shanghai Chest Hospital.

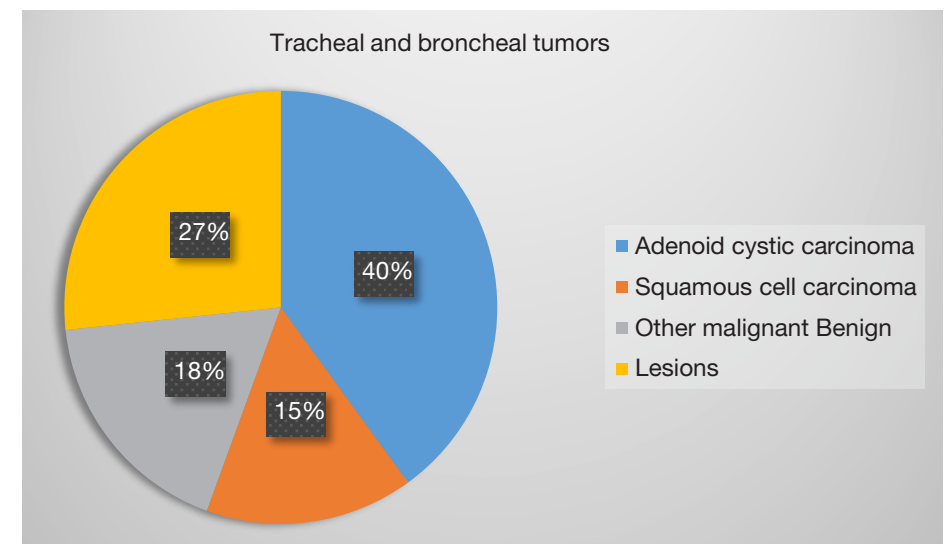

Figure 24 Histology of tracheal and bronchial tumors in Shanghai Chest Hospital in 2018.

invasive thoracic procedures, benefiting hundreds of residents and fellows.

\section{Discussion}

Since 2017, the thoracic surgery team of Shanghai Chest Hospital has released the annual report to public.
Similar to the previous version, this report systematically summarized the key achievements made in the past year by the Shanghai Chest thoracic surgery team. As one of the largest thoracic disease centers in the world, Shanghai Chest has accumulated ample clinical experience to treat all kinds of complex, difficult and severe chest diseases. Services at the Shanghai Chest could be taken as an 


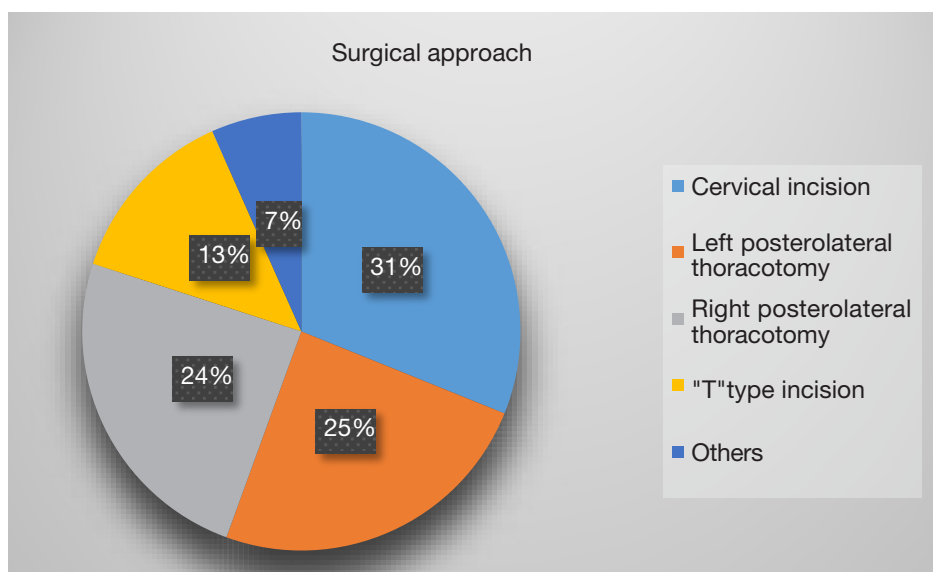

Figure 25 Surgical approaches of trachea disease carried out in Shanghai Chest Hospital in 2018.
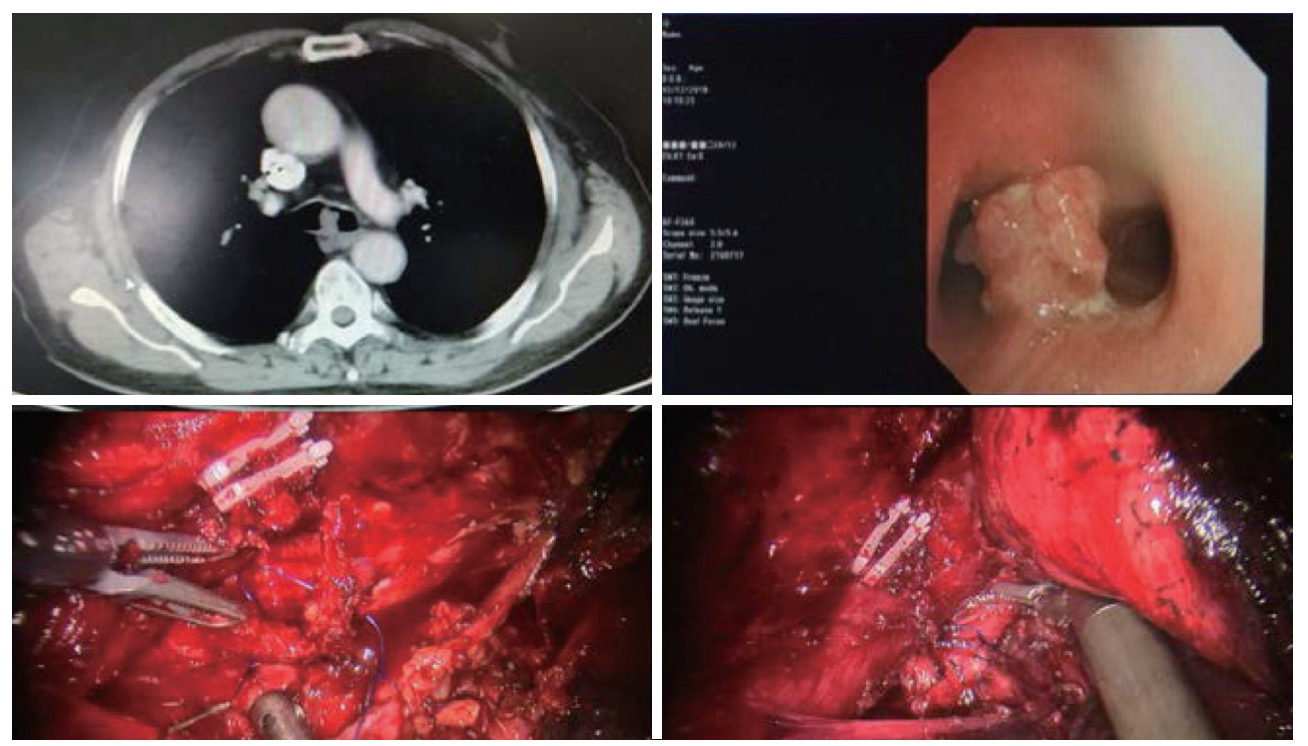

Figure 26 Preoperative examinations and inoperative situation of robotic assisted carina resection and reconstruction.

epitome of thoracic surgery in China. In 2018, surgical volume exceeded 10,000 cases for 3 consecutive years. In the meantime, rates of unplanned reoperation, readmission to ICU, and 30-day mortality have been curtailed to an impressively low level. Reporting these satisfying results is not only important for the interest of peers and patients to have a better understanding about the quality of thoracic surgical services in China, but also for self-improvement in the future. As a recognized leader in the field of thoracic diseases, Shanghai Chest should be proficient in the surgical treatment of difficult and complicated surgical thoracic diseases, and implement cutting-edge technologies to lead the development of thoracic surgery. This report would serve as a valuable reference and provide useful information to thoracic surgeons both home and abroad.

Lung cancer is the leading cause of cancer death in China. With increasing use of CT screening, there has been a rapid increase in incidental detection of small pulmonary nodules. Although the results of the NLST showed a $20 \%$ improvement in survival by CT screening, there was also an $18 \%$ untherapeutic surgery for misdiagnosed benign lesions. Remarkably, diagnostic accuracy at the Shanghai Chest Hospital was as high as $91.3 \%$ in 2018 , owing to careful clinical evaluation 
and stringent surgical indication. Also because of CT screening, sublobar resections have been increasingly applied for small GGO containing lesions. It is noteworthy that number of segmentectomy reached 1,321 in 2108, increased by $36.2 \%$ from 2017 , almost all of them were accomplished via minimally invasive approaches. This would certainly help improve surgical recovery and quality of life in patients with early stage lung cancers in China.

In addition to surgery for early stage diseases, there is also a remarkable increase in using minimally invasive surgical techniques to fulfill complicated pulmonary procedures for locally advanced diseases. Thoracic Surgery service at the Shanghai Chest Hospital has a well-established reputation in complex pulmonary procedures. Together with the advent in minimally invasive surgery in the last 20 years, surgery for centrally located lung cancers as well as those with locally advanced diseases needing induction therapies, is now increasingly carried out under VATS/ RATS. Noticeably, among a total of 119 sleeve resections in $2018,25 \%$ of them were successfully done by VATS. What is more, 16 cases of VATS (18\%) was witnessed in a total of 87 pneumonectomies. Although minimally invasive sleeve resections and pneumonectomies are technically demanding even in open surgery, the increasing use of VATS and RATS for these complex procedures at the Shanghai Chest represents a marked step forward in surgical management of locally advanced lung cancers. More patients are thus expected to benefit from the advent of surgical techniques in the future.

A similar tread in increased use of minimally invasive approaches has also been seen in esophageal surgery at the Shanghai Chest, with over $80 \%$ of esophagectomies for malignant lesions accomplished by thoracoscopic/ laparoscopic or robotic surgery. In addition to ESD for early stage esophageal cancers, novel techniques such as single-port mediastinoscopic esophagectomy has been a new highlight in this field. All these efforts world help diminish the significant trauma caused by esophagectomy as an extensive and devastating procedure for already fragile esophageal cancer patients. Meanwhile, tumors located higher up at the cervical-thoracic junction pose significant challenge to esophageal surgeons. Tremendous efforts have been implemented at the Shanghai Chest to assure radical removal of the disease while preserving functions to the greatest extent. These would include TPLE, LPE, as well as free jejuna interposition. With long-term experience accumulated by generations of surgeons, rates of mortality and morbidity remains at acceptable levels even with rapid increase in difficult cases including patients with locally advanced diseases and those after induction therapies aiming at improving therapeutic outcomes.

The Mediastinal Surgery Division has made great endeavour to implore minimally invasive surgical techniques into surgical management of locally invasive tumors (UICC 8th TNM staging II-III). This could be seen from the rapid increase in number of complex anterior mediastinum procedures accomplished under VATS since 2010. Noticeably, three patients with locally advanced thymic tumors invading superior vena cava underwent thoracoscopic thymectomy with tangential superior vena cava resection, which had not been reported before.

The continuous increase in number of complicated anterior mediastinum procedures via open surgery represents the ability to treat locally advanced TETs at the Shanghai Chest Hospital. Nearly $40 \%$ of these patients received preoperative induction therapies. The concomitant increase in use of induction therapy and the increase in complex surgeries demonstrates the continuously improving ability to manage difficult mediastinal disorders at the Shanghai Chest Hospital. All these have become possible only with incessant endeavours in clinical researches including the feasibility study of minimally invasive surgery for locally advanced TETs and induction sequential chemoradiation for potentially resectable stage IIIb-IV diseases. Also with ongoing trials on surgical pleurectomy followed by hemi-thorax pleural irradiation for stage IVa TETs and the phase II trial for a domestically developed novel anti-VEGF agent, achievements could also be expected in difficult conditions related to late stage/ recurrent thymic tumors. Hopefully the results of these trials would significantly improve the management of this relatively rare disease.

Airway resection and reconstruction is often technically demanding and requires the ability to perform various different surgical approaches. Shanghai Chest Hospital has been the leading center in tracheal surgery in China since its establishment in the 1950's. Individualized surgical approach for optimal exposure is the key point for successful airway reconstruction after resection of the lesion. And with the help of extra-corporal circulation team, tracheal surgery has become a safe procedure even in case of difficult airways. Similar to pulmonary, esophageal, and mediastinal surgery at the Shanghai Chest, minimally invasive techniques have started to play important roles in airway resection and reconstructions. The first successful carinal resection by RATS is just 
another excellent example. And with increasing experience in difficult thoracic procedures, it may be foreseeable that sooner or later minimally invasive techniques would be incorporated into other major procedures, even including lung transplantations.

The rapid surge in surgical volume at Shanghai Chest provides unprecedented resources for clinical researches as well as training opportunities for young thoracic surgeons. In fact, such clinical resources are not only helpful for studies of common chest diseases such as lung and esophageal cancers, they are even more precious for relatively rare diseases such as mediastinal malignancies. Major progress could thus be expected when such resources could be fully utilized.

In ultra-high volume centers where up to a hundred major procedures are ongoing on the daily base, shorter period intensive clinical training may offer equivalent or even better education than months or years training at traditional high-volume centers. And more versatile approaches may be observed with dozens of surgeons operating simultaneously. These clinical education activities rely heavily on the joint effort by the hospital and the industries. Realizing the increasing demand for such training, the Shanghai Chest is going to set up its own simulation center and a wet lab inside the hospital to facilitate future training programs.

\section{Conclusions}

This annual report reviewed work and efforts made in 2018 in thoracic surgery service at the Shanghai Chest Hospital. In addition to continuous sharp increase in surgical volume, remarkable achievements have been accomplished in high quality care and novel techniques. With the aim of providing optimal clinical care, creative surgical innovation,

doi: 10.21037/shc.2019.12.07

Cite this article as: Wang Y, Luo J, Yang Y, Cheng X, Chen T, Yao F, Gu Z, Sun Y, Luo Q, Li Z, Yang J, Mao T, Fu S, Wang R, Zhang J, Zhao H, Yu Y, Pan C, Fang W. 2018 annual report of thoracic surgery service at Shanghai Chest Hospital. Shanghai Chest 2019;3:70. and first-rate education, the Thoracic Surgery team at the Shanghai Chest will strive to deliver higher levels of medical care and will spare no effort in related research and education so as to ultimately benefit our patients.

\section{Acknowledgments}

Funding: None.

\section{Footnote}

Conflicts of Interest: All authors have completed the ICMJE uniform disclosure form (available at http://dx.doi. org/10.21037/shc.2019.12.07). Wentao Fang serves as an unpaid Executive Editor-in-Chief of Shanghai Chest. The other author has no conflicts of interest to declare.

Ethical Statement: The authors are accountable for all aspects of the work in ensuring that questions related to the accuracy or integrity of any part of the work are appropriately investigated and resolved. This study was conducted in accordance with the Declaration of Helsinki (as revised in 2013). The institutional ethical approval and individual informed consent were waived.

Open Access Statement: This is an Open Access article distributed in accordance with the Creative Commons Attribution-NonCommercial-NoDerivs 4.0 International License (CC BY-NC-ND 4.0), which permits the noncommercial replication and distribution of the article with the strict proviso that no changes or edits are made and the original work is properly cited (including links to both the formal publication through the relevant DOI and the license). See: https://creativecommons.org/licenses/by-nc-nd/4.0/. 


\section{Supplementary file 1}

\section{Ongoing Clinical and Laboratory Research Projects in 2018}

1. Wenyong Zhou. Establishment of experimental animal model of chronic rejection of vascularized orthotopic lung transplantation in mice. Sub topics of experimental animal research project of Shanghai 2018 "action plan of scientific and technological innovation".

2. Bo Ye. Clinical application of artificial intelligence recognition technology in the diagnosis of early lung cancer. Shanghai transformation medicine Collaborative Innovation Center Project.

3. Zhigang Li. Detection of sentinel lymph nodes in esophageal cancer by fluorescence tracing with indocyanine green. Shanghai transformation medicine Collaborative Innovation Center Project.

4. Wentao Fang. Prospective multicenter randomized controlled clinical study of adjuvant radiotherapy after radical thymectomy for high-risk thymic tumors. Multicenter clinical research project of Medical College of Shanghai Jiaotong University.

5. Wentao Fang. Accurate typing of thymic tumors based on quantitative proteomics of DIA. 2018 key project of "transforming medical cross research fund" of Jiaotong University.

6. Rong Hua. Functional study of DNA replication sequence, genetic diversity of p53 gene family, susceptibility and prognosis of esophageal cancer. Class B youth project of "cross research fund for translational medicine" of Jiaotong University in 2018.

7. Wentao Fang. Grading treatment of advanced thymic tumor under the guidance of multi-dimensional histology. Clinical research project of multi-disciplinary collaborative innovation in hospital in 2018 - major project.

8. Zhigang Li. Evaluation and diagnosis of modular clinical examination after neoadjuvant radiotherapy and chemotherapy for esophageal squamous cell carcinoma. 2018 hospital multidisciplinary collaborative innovation clinical research project - Key Cultivation Project.

9. Zhigang Li. Robot-assisted Esophagectomy Versus Conventional Thoracoscopic Esophagectomy for Patients With Squamous Cell Esophageal Cancer: a Multicenter Open-label, Randomized Controlled Trial (RAMIE Trial).

10. Zhigang Li. Accuracy of Detecting Residual Disease After Neoadjuvant Chemoradiotherapy for Esophageal
Squamous Cell Carcinoma: the preSINO Trial (PreSurgery If Needed for Oesophageal Cancer).

11. Zhigang Li. Prospective, Single-arm, and Exploratory Phase II Clinical Study for the Efficacy of Apatinib in the Treatment of Recurrence or Metastasis of Esophageal Squamous Cell Carcinoma After Radical Resection.

12. Zhigang Li. Adjuvant Esophagectomy Versus Chemoradiation for Patients With Clinical Stage $\mathrm{N} 0$ and Pathological Stage T1b (After Endoscopic Submucosal Dissection) Esophageal Squamous Cell Carcinoma: a Multicentric, Open Label, Randomized Trial (Ad-ESD Trial).

13. Feng Yao. Optimization of precise surgical treatment strategy for early lung cancer under the guidance of multi-dimensional histology. 2018 hospital multidisciplinary collaborative innovation clinical research project - Key Cultivation Project.

14. Xufeng Guo. A prospective, randomized, controlled study of robotic minimally invasive surgery and traditional open surgery after neoadjuvant concurrent radiotherapy and chemotherapy for locally advanced esophageal cancer. Wu Jieping medical fund.

15. Bo Ye. Research on theory and application of adaptive detection and reconstruction of 3D hidden boundary surface. National Nature project of Shanghai Jiaotong University.

16. Wentao Fang. Individualized study of clinical antitumor and autoimmune drugs. Special project of strategic leading science and technology of Chinese Academy of Sciences.

\section{Supplementary file 2}

\section{Publications in 2018}

1. Pan X, Gu C, Yang J, et al. Robotic double-sleeve resection of lung cancer: technical aspects. Eur J Cardiothorac Surg 2018;54:183-4.

2. Wang Y, Zheng D, Zheng J, et al. Predictors of recurrence and survival of pathological T1N0M0 invasive adenocarcinoma following lobectomy. J Cancer Res Clin Oncol 2018;144:1015-23.

3. Fang W, Wang Y, Pang L, et al. Lymph node metastasis in thymic malignancies: A Chinese multicenter prospective observational study. J Thorac Cardiovasc Surg 2018;156:824-33.e1.

4. Gu Z, Wang H, Mao T, et al. Pulmonary function 
changes after different extent of pulmonary resection under video-assisted thoracic surgery. J Thorac Dis 2018;10:2331-7.

5. Yuan Y, Ma G, Zhang Y, et al. Presence of micropapillary and solid patterns are associated with nodal upstaging and unfavorable prognosis among patient with cT1N0M0 lung adenocarcinoma: a large-scale analysis. J Cancer Res Clin Oncol 2018;144:743-9.

6. Huang Q, Wang R, Gu C, et al. Appropriate lymphadenectomy significantly reduced recurrence after segmentectomy for patients with non-small cell lung cancer. J Thorac Dis 2018;10:1919-26.

7. Gu C, Pan X, Chen Y, et al. Short-term and mid-term survival in bronchial sleeve resection by robotic system versus thoracotomy for centrally located lung cancer. Eur J Cardiothorac Surg 2018;53:648-55.

8. Zhong C, Sakurai H, Wei S, et al. Sublobar resections for small-sized stage Ia lung adenocarcinoma: a SinoJapanese multicenter study. J Thorac Dis 2018;10:991-8.

9. Guo X, Ye B, Yang Y, et al. Impact of unplanned events on early postoperative results of minimally invasive esophagectomy. Thorac Cancer 2018;9:94-8.

10. Yang H, Liu H, Chen Y, Zhu C, Fang W, et al. Neoadjuvant Chemoradiotherapy Followed by Surgery Versus Surgery Alone for Locally Advanced Squamous Cell Carcinoma of the Esophagus (NEOCRTEC5010): A Phase III Multicenter, Randomized, Open-Label Clinical Trial. J Clin Oncol 2018;36:2796-803.

11. Zhang X, Gu Z, Fang W. Minimally invasive surgery in thymic malignances: the new standard of care. J Thorac Dis 2018;10:S1666-70.

12. Mei X, Wang R, Yang W, et al. Predicting malignancy of pulmonary ground-glass nodules and their invasiveness by random forest. J Thorac Dis 2018;10:458-63.

13. Gu Z, Chen C, Wang Y, et al. Video-assisted thoracoscopic surgery versus open surgery for Stage I thymic epithelial tumours: a propensity score-matched study. Eur J Cardiothorac Surg 2018;54:1037-44.

14. PanX, YangJ, Fu S, et al. Application of ex vivo lung perfusion (EVLP) in lung transplantation. J Thorac Dis 2018;10:4637-42.

15. Huang Q, Zhang H, Hai J, et al. Impact of PD-L1 expression, driver mutations and clinical characteristics on survival after anti-PD-1/PD-L1 immunotherapy versus chemotherapy in non-small-cell lung cancer: A meta-analysis of randomized trials. Oncoimmunology 2018;7:e1396403.

16. Guo X, Fang W, Li Z, et al. Adjuvant radiotherapy, chemotherapy or surgery alone for high-risk histological node negative esophageal squamous cell carcinoma: Protocol for a multicenter prospective randomized controlled trial. Thorac Cancer 2018;9:1801-6.

17. Li ZG, Zhang XB, Wen YW, et al. Incidence and Predictors of Unsuspected Recurrent Laryngeal Nerve Lymph Node Metastases After Neoadjuvant Chemoradiotherapy in Patients with Esophageal Squamous Cell Carcinoma. World J Surg 2018;42:2485-92.

18. Yang J, Pan X, Fu S, et al. Pulmonary retransplantation. J Thorac Dis 2018;10:4632-6.

19. Zhang X, Su Y, Yang Y, et al. Robot assisted esophagectomy for esophageal squamous cell carcinoma. J Thorac Dis 2018;10:3767-75.

20. Li B, Yang Y, Sun Y, et al. Minimally invasive esophagectomy for esophageal squamous cell carcinoma-Shanghai Chest Hospital experience. J Thorac Dis 2018;10:3800-7.

21. Shen Y, Li W. HA/HSA co-modified erlotinib-albumin nanoparticles for lung cancer treatment. Drug Des Devel Ther 2018;12:2285-92.

22. Chen C, Wang Y, Fu S, et al. The impact on mediastinal recurrence based on the number of harvested mediastinal lymph nodes and assessed N2 Stations in patients with stage I invasive lung adenocarcinoma. J Thorac Dis 2018;10:6803-10.

23. Chen C, Wang Y, Pan X, et al. Choice of the surgical approach for patients with stage I lung squamous cell carcinoma $\leq 3 \mathrm{~cm}$. J Thorac Dis 2018;10:6771-82.

24. Zhang S, Ma Y, Hu X et al. Targeting PRMT5/Akt signaling axis prevents human lung cancer cell growth. J Cell Mol Med 2019;23:1333-42.

25. Shang Z, Qian L, Liu S, et al. Graphene Oxidefacilitated comprehensive analysis of cellular nucleic acid binding protein foe lung cancer. ACS Appl Mater Interfaces 2018;10:17756-70.

26. Huang J, Li J, Li H, et al. Continuous 389 cases of $\mathrm{Da}$ Vinci robot-assisted thoracoscopic lobectomy in treatment of non-small cell lung cancer: experience in Shanghai Chest Hospital. J Thorac Dis 2018;10:3776-82.

27. Li Z, Huang J, Shen S, et al. SIRT6 drives epithelialto-mesenchymal transition and metastasis in non-small cell lung cancer via snail-dependent transrepression of KLF4. J Exp Clin Cancer Res 2018;37:323.

28. Li H, Li J, Huang J, et al. Robotic-assisted mediastinal surgery: the first Chinese series of 167 consecutive cases. J Thorac Dis 2018;10:2876-80. 
29. Li H, Hu Y, Huang J, et al. Attempt of peripheral nerve reconstruction during lung cancer surgery. Thorac Cancer 2018;9:580-3.

30. Cheng X, Li C, Huang J, et al. Three-arm robot-assisted thoracoscopic surgery for locally advanced N2 non-small cell lung cancer. J Thorac Dis 2018;10:7009-13.

31. Chen T, Luo J, Gu H, et al. Should minimally invasive lung adenocarcinoma be transferred from stage IA1 to stage 0 in future updates of the TNM staging system? J Thorac Dis 2018;10:6247-53.

32. Chen T, Luo J, Wang R, et al. Prognosis of limited resection versus lobectomy in elderly patients with invasive lung adenocarcinoma with tumor size less than or equal to $2 \mathrm{~cm}$. J Thorac Dis 2018;10:2231-9.

33. Chen T, Luo J, Gu H, et al. Impact of Solid Minor Histologic Subtype in Postsurgical Prognosis of Stage I Lung Adenocarcinoma. Ann Thorac Surg 2018;105:302-8.

34. Liu R, Tan Q, Luo Q. Decreased expression level and DNA-binding activity of specificity protein 1 via cyclooxygenase-2 inhibition antagonizes radiation resistance, cell migration and invasion in radiationresistant lung cancer cells. Oncol Lett 2018;16:3029-37.

\section{Supplementary file 3}

\section{Education Events and Training Programs in 2018}

As efforts for continuing medical education, the following conferences were hosted by the Shanghai Chest Hospital in 2018:

(I) The $9^{\text {th }}$ shanghai International Lung Cancer Forum and the Fifth China-Switzerland International Lung Cancer Forum;

(II) Shanghai Chest hospital $2^{\text {nd }}$ International Summit on Esophageal Surgery;

(III) The $2^{\text {nd }}$ seminar on Gastroesophageal Reflux Disease and Benign Esophageal Diseases;

(IV) The $1^{\text {st }}$ China-Eastern Europe International Forum on Thoracic Diseases. 